\title{
An approach for human gait identification based on area
}

\author{
Jasvinder Pal Singh ${ }^{1}$, Sanjay Agrawal ${ }^{2}$ \\ ${ }^{I}$ Department of Computer Science and Engineering, RKDFIST, Bhopal, India \\ ${ }^{2}$ Department of Computer Engineering and Applications, NITTTR, Bhopal, India
}

\begin{abstract}
In recent investigations related to security issues, biometrics plays an important role in recognition of individuals based on their physiological or behavioral characteristics. Gait as a biometric behavioral trait plays an important role in recent research. Gait is an unobtrusive biometric, is the process of identifying an individual by the manner in which they walk. In this paper we propose a concept of geometric for human gait recognition. We have considered dynamic features and side view of human body for gait recognition. This paper considers three dynamic parameters i.e right hand, left feet and right feet. Then a triangle is formulated between these parameters and computed area for different subjects and store it in database for recognition.
\end{abstract}

Keywords: Biometrics, Gait recognition, triangle.

\section{Introduction}

Biometrics recognition systems are originated from real life criminal and forensic applications. Some methods such as finger prints and face recognition already proved to be very efficient in human recognition [1].Biometrics is a combination of "Bio" means life and "metrics" means measure [10]. Biometrics is defined as the science and technology of measuring and analyzing biological aspects of human being. Biometrics Technologies are classified into two categories shown in Fig.1:

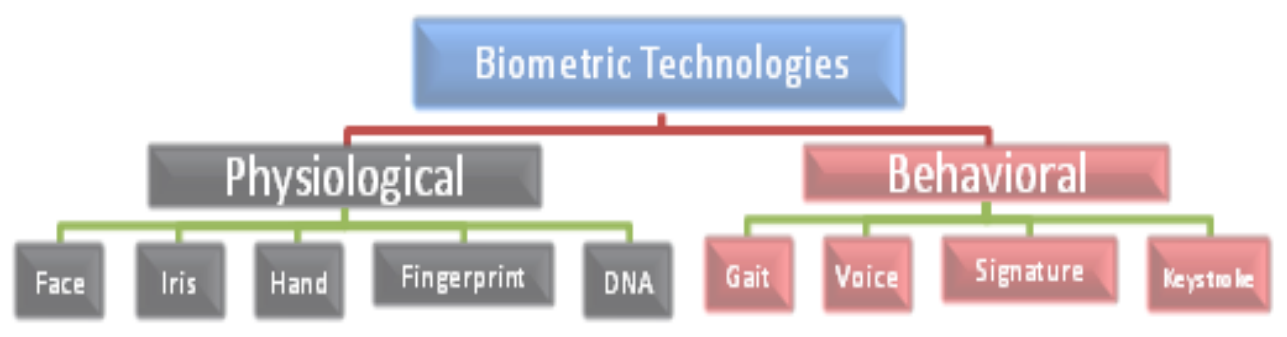

Figure 1. Classification of biometrics Technologies

Physiological Biometrics: these are biometrics which is derived from a direct measurement of a part of human body. The most prominent and successful of these types of measures to date are finger prints, face recognition, iris scans and hand scans. Behavioral Biometrics: extract characteristics based on an action performed by an individual. Established measure includes key-stroke scan and speech patterns. In this paper, we present a behavioral biometrics recognition system based on Gait [5]. Vision based human identification at a distance in surveillance applications have recently gained more importance. Gait recognition [6] as a new behavioral biometrics aims at recognizing individuals by the way they walk. In comparison with other biometrics such as finger prints and iris, gait has the advantage of being non-invasive and noncontact and it is also the only perceivable biometrics for personal identification [2][4][10]. Biometric gait recognition method mainly divided into two categories: model based and motion based models. In model based approach , the human body structure or motion is modeled first and then the image features are extracted by measuring the structural components of models or by measuring the motion trajectories[6][11]. Motion based approach is further classified into statespace methods and spatiotemporal methods [12][13]. The state- space methods consider gait motion to be composed of a sequence of static body poses and recognize it by considering temporal variation observations. The spatiotemporal method characterizes the spatiotemporal distribution by collapsing the entire 3D spatiotemporal data over an entire sequence into 1D or 2D signals [13].

\section{Proposed Work}

Biometric gait is a behavioral biometric. Human body is divided into static and dynamic body parameters [5]. We have considered dynamic body parameters. Our proposed work is divided into two blocks i.e preprocessing and post processing as shown in Fig.2. 


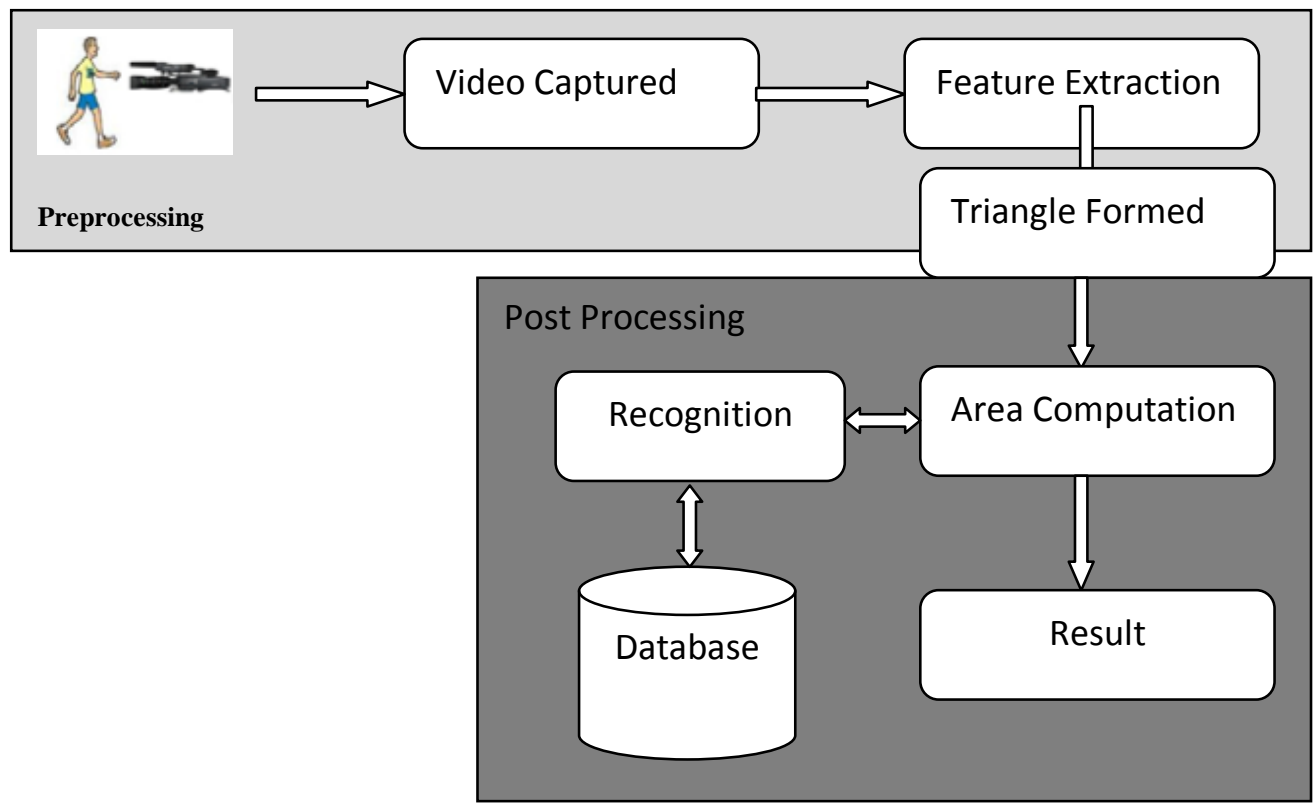

Figure 2. Block Architecture of Proposed work.

In biometric system data to be collected is in video format. So, in preprocessing initially video of a particular person is captured, and then this captured video is into converted frames of that particular person. In our work we have considered side view of particular subjects. Here we have considered three parameters of the human body for feature extraction. i.e Left hand, right feet and left feet. Total three control points are formed and a dot point is inserted on these control points of an individual subject as shown in Fig.3.

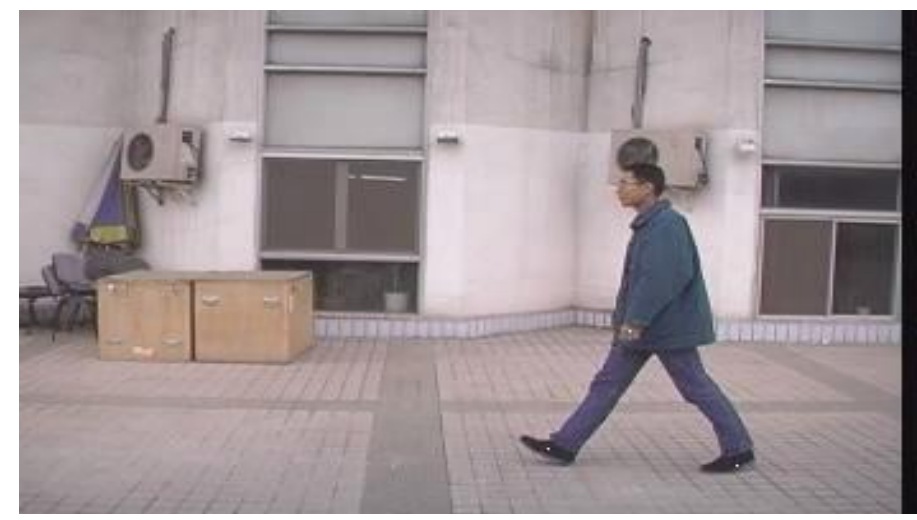

Figure 3.side view of particular person with dot point on three parameters (Left hand, Right feet and left feet).

Then a single triangle is formed on these control points using the proposed gait system that is designed in Matlab R2011a for gait recognition. The triangle is drawn between left hand, right feet and left feet as described in Fig.4.

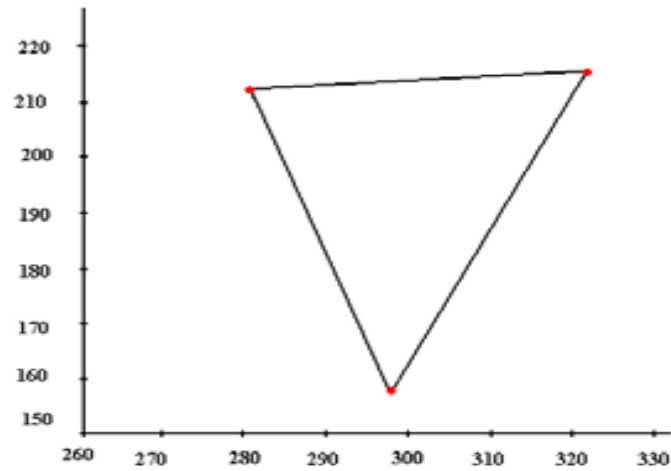

Figure 4. Triangle formed on three control points. 
Our main motive of triangle formulation is to compute area of triangle. Area of triangle is computed by using Heron's Formula [8][9]. In geometry Heron's formula or Hero's formula states that the area T of a triangle whose sides have length a, b, c refer Fig.5 with vertex(x1, y1), (x2, y2) and (x3, y3) is computed as

$T=\sqrt{s(s-a)(s-b)(s-c)}$

Here $\mathrm{s}$ is a semiperimeter, which is calculated as $s=\frac{a+b+c}{2}$

In our proposed work, length of sides of triangle i.e a, b, c as shown in Fig.5.is computed using distance method [9].

$a=\sqrt{(x 2-x 1)^{2}+(y 2-y 1)^{2}}$

$b=\sqrt{(x 3-x 2)^{2}+(y 3-y 2)^{2}}$

$c=\sqrt{(x 3-x 1)^{2}+(y 3-y 1)^{2}}$

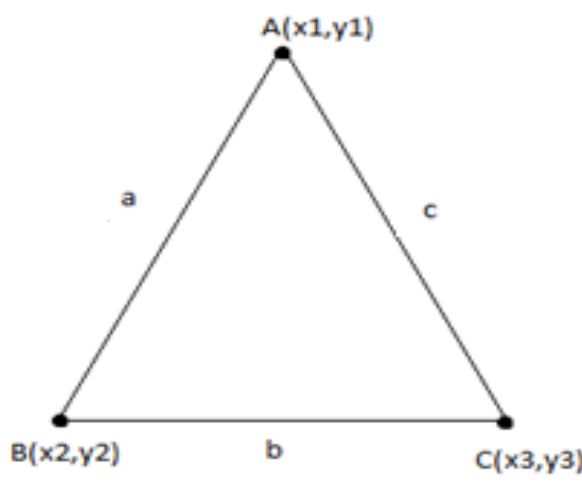

Figure 5. Shows triangle formed on three control points with sides a, b, c.

Table I shows an example of a particular person triangle sides length with multiple frames.

\begin{tabular}{|c|c|c|c|}
\hline Frames & Distance a & Distance b & Distance c \\
\hline 1 & 52.2015 & 44.1022 & 79.3095 \\
\hline 2 & 51.6624 & 44.4072 & 79.9062 \\
\hline 3 & 49.8197 & 44.9110 & 78.5684 \\
\hline 4 & 48.3735 & 44.1475 & 75.9276 \\
\hline 5 & 41.4367 & 39.3954 & 70.8025 \\
\hline 6 & 42.2019 & 26.9258 & 64.1405 \\
\hline 7 & 44.6430 & 16.5529 & 50.0899 \\
\hline 8 & 50.2892 & 28.0713 & 51.6624 \\
\hline 9 & 49.9800 & 42.2966 & 57.6975 \\
\hline 10 & 51.5461 & 51.2445 & 64.3506 \\
\hline 11 & 55.7315 & 54.0093 & 71.8401 \\
\hline 12 & 75.743 & 57.1402 & 60.2993 \\
\hline 13 & 79.9312 & 53.1507 & 62.0322 \\
\hline 14 & 77.0779 & 46.8722 & 63.6396 \\
\hline 15 & 68.6222 & 40.2616 & 60.5310 \\
\hline 16 & 58.8218 & 28.1603 & 56.4358 \\
\hline 17 & 50.1597 & 17.0294 & 55.1543 \\
\hline 18 & 65.4370 & 32.0156 & 52.7731 \\
\hline 19 & 76.4199 & 46.0109 & 53.9351 \\
\hline 20 & 81.5659 & 54.0370 & 55.5788 \\
\hline
\end{tabular}

The table I shows an example of values of triangle sides a,b,c of a particular subject frames by using equation $3,4 \& 5$.Then Area of triangle for a particular person frames are computed from equation 1 , an example shown in Fig 6. 


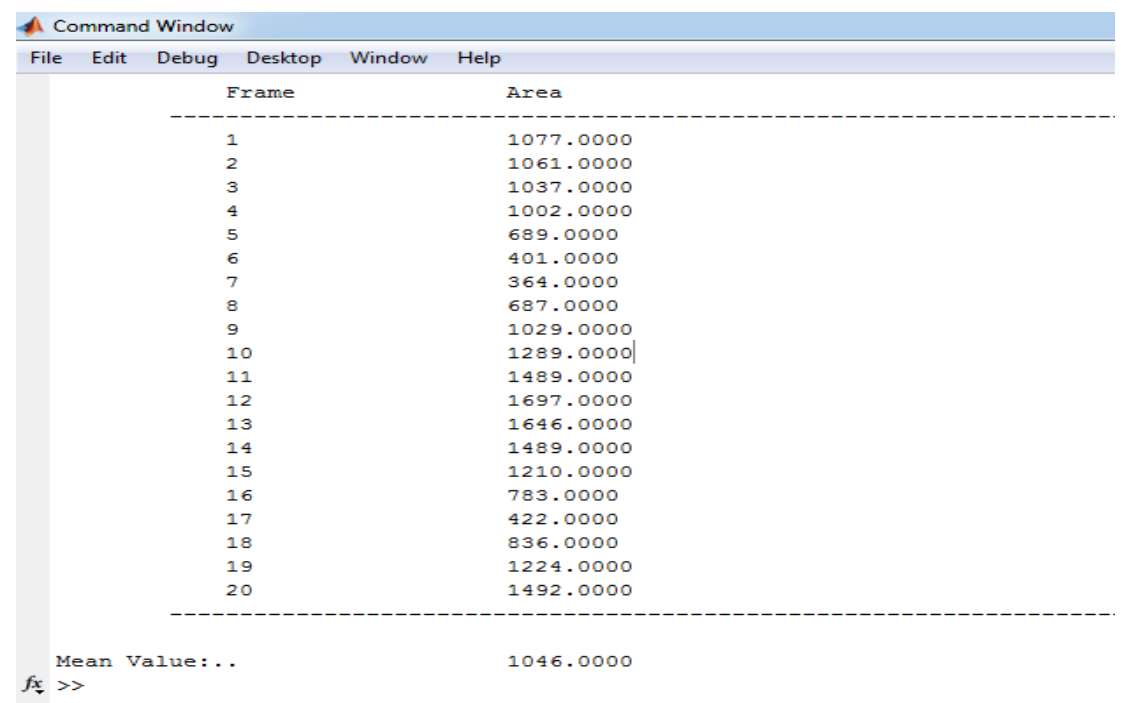

Figure 6. Area of multiple frames of a particular subject.

Then a mean is calculated of multiple frame areas of an individual subject. That mean value is stored in database for recognition purpose. These steps are repeated for next subject frames.

1. Input subject gait cycle frames.

\section{Proposed Algorithm}

2. Select parameters for feature extraction :

a) left hand

b) Left feet

c) Right feet

3. Construct triangle between these features.

4. Compute area of triangle on subject gait frames.

5. Compute mean of calculated areas of multiple frames of an individual subject and store in database.

6. Repeat step 1 to 5 for next subject gait cycle frames.

7. Classify result in the database.

\section{Experiments And Result Analysis}

The CASIA gait dataset A [7] have been used to analyze the effectiveness of the proposed work. In CASIA gait dataset A, frames of individual subject are a format of *.png and with resolution of $352 * 240$. The database consists of 17 subjects.

Table II. Area mean value of individual subjects.

\begin{tabular}{|c|c|}
\hline Subjects & Area Mean Value \\
\hline A & 1046 \\
\hline B & 1316 \\
\hline C & 1325 \\
\hline D & 1695 \\
\hline E & 2662 \\
\hline F & 1403 \\
\hline G & 1138 \\
\hline H & 1334 \\
\hline I & 1204 \\
\hline J & 1519 \\
\hline K & 1046 \\
\hline L & 2482 \\
\hline M & 1527 \\
\hline
\end{tabular}




\begin{tabular}{|c|c|}
\hline $\mathrm{N}$ & 1563 \\
\hline $\mathrm{O}$ & 1248 \\
\hline $\mathrm{P}$ & 1886 \\
\hline $\mathrm{Q}$ & 1662 \\
\hline
\end{tabular}

Table II shows the mean value of area of triangle of each individual subjects. Form the result we have analyze that subject $\mathrm{A}$ and subject $\mathrm{K}$ have same area and the average classification rate we achieve is $88 \%$.

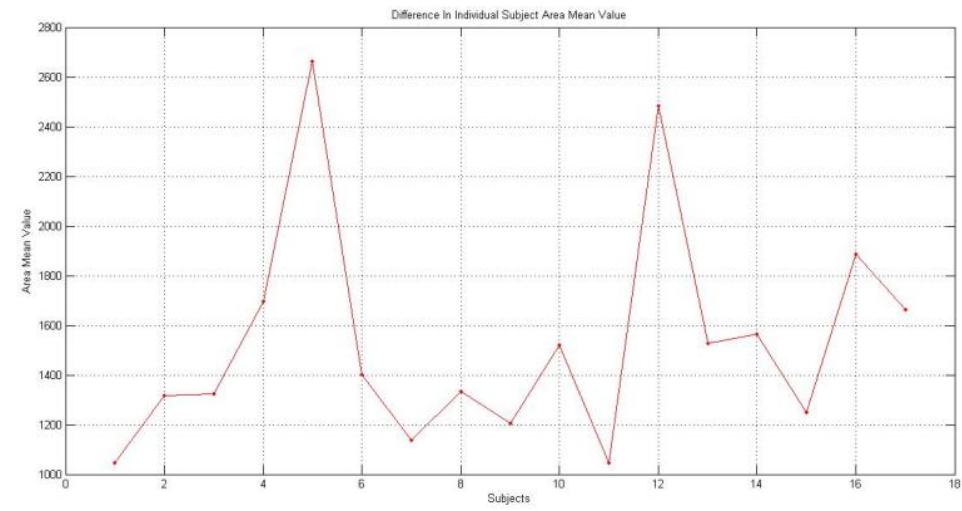

Figure 7 Shows result of proposed algorithm.

The Fig.7 shows the difference in area mean value of each subject. $X$ axis represent number of subjects and $y$ axis represent area mean value.

\section{Conclusion}

This paper present gait recognition approach using geometry concept of triangle. Triangle is formed on three control point's left hand, right feet and left feet and calculate area of triangle as a feature for recognition. The average recognition rate we achieved is $88 \%$ as compared to other algorithms [5].

\section{References}

[1] Michal Choras,Emerging Methods of biometrics human identification, 2nd International Conference on Innovative Computing, Information and Control, ICICIC '2007, pp365 - 365 .

[2] Liang Wang, Weiming Hu, Tieniu Tan,A new attempt to Gait-based Human Identification, the 16th IEEE international conference on pattern recognition ICPR2002, 10 Dec 2002,pp 115 - 118

[3] Mark S.Nixon,John N.Carter,Advances in automatic gait recognition, Sixth IEEE International Conference on Automatic Face and Gesture Recognition, 2004, pp139-144.

[4] Xuelong li, Stephen J.Maybank, Shuicheng Yan,Dacheng Tao and Dong Xu ,Gait components and their applications to Gender recognition, IEEE Transactions on Systems, Man, and Cybernetics, Part C: Applications and Reviews 2008, pp 145 - 155.

[5] Jasvinder pal singh and Sanjeev jain, Person Identification Based on Gait using Dynamic Body Parameters, IEEE International Conference on Trendz in Information Sciences \& Computing (TISC) 2010,pp: 248 - 252 .

[6] Nikolaos V.Boulgouris,Dimitrious Hatzinakos and Konstantinos N.Plataniotis, Gait Recognition: A challenging signal processing technology for biometric identification ,IEEE signal Processing Magazine, November 2005, vol.22,No.6,pp78-90.

[7]. www.cbsr.ia.ac.cn

[8] http://en.wikipedia.org/wiki/Heron's formula

[9] Dr.B.S.Grewal, higher engineering mathematics, khanna Publishers ed.40 ${ }^{\text {th }}$ ISBN 9788174091956.

[10] Anil K. Jain, Arun Ross, and Sharath Pankanti,Biometrics: A Tool for Information Security, IEEE Transactions on Information Forensics and Security, June 2006, pp 21-38.

[11] C.Y.Yam, M.S.Nikon and J.N.Carter, Gait recognition by walking and running: A model based approach, Proceeding of $5^{\text {th }}$ Asian Conference on computer Vision, 2002, pp.1-6.

[12] C.BenAbdelkader,R.Culter,H.Nanda and L.Davis,EigenGait: Motion Based Recognition of People using Image Self-Similarity, Proceeding international conference Audio and Video based Biometric Person authentication,2001,pp.284-294.

[13] L.R Sudha, Dr.R.Bhavani, Biometric Authorization system Using Gait Biometry, International Journal of Computer Science,Engineering and Applications(IJCSEA),August 2011,Vol.1,No.4. 